\title{
2006-1290: QUASI INTERACTIVE VIDEO: AN INNOVATIVE APPROACH TO THE DELIVERY OF LABORATORY CLASSES
}

\section{Peter Burton, Royal Melbourne Institute of Technology}

Peter C. M. Burton is Senior Lecturer in the School of Electrical and Computer Engineering at RMIT. He has also taught extensively and worked in the microelectronics industry in the United States. A background and strong interest in video production, coupled with an appreciation of difficulties faced by some first year tertiary students has been the impetus for this current work.

\section{Sanjay Kumar, Royal Melbourne Institute of Technology}

Sanjay Kumar is a Research Associate in the School of Electrical and Computer Engineering. His background is in biomedical engineering and computer vision. He is currently working on video production with Peter Burton.

Dinesh Kumar, Royal Melbourne Institute of Technology 


\title{
Quasi Interactive Video: An Innovative Approach to the Delivery of Laboratory Courses
}

\begin{abstract}
Quasi interactive video material has proved to be a very effective teaching tool for first year electronic classes at RMIT University and has been the subject of a previous ASEE paper and presentation $^{[1]}$.

Quasi interactive video material at RMIT is offered as an addition to and not instead of regular lecture classes and takes its name from the fact that lectures are not merely filmed but the videos are scripted, filmed and produced in a manner that makes the student feel that he or she is actively engaging with the presenter.
\end{abstract}

Building on the success of the quasi interactive videos for first year lecture classes and in an effort to overcome many of the problems associated with laboratory instruction, the authors set out to undertake a pilot project to see if quasi interactive video could also be introduced to aid student learning in the traditionally challenging area of laboratory teaching.

This paper outlines the pilot approach taken and the rationale for lab video production. It also explains the lab structure and the video production methods used. Feedback was sought from students and laboratory instructors and this is discussed. Finally the paper outlines the many benefits and advantages in producing a test series of quasi interactive videos to supplement laboratory teaching in first year electronics, which include more uniform quality and consistency across all lab sections, as well as the opportunities for use in distance education.

Introduction

The continued success of video material for the first year electronics course at RMIT University has prompted video development in other courses as well as development for promotional purposes.

Since video material first became available at RMIT, students have been consistently requesting videos for other courses and also for laboratories. The laboratory has often proved to be the most difficult and daunting course, particularly for first year students, and there are a number of reasons why this is the case.

The laboratory environment is quite different to the lecture theatre and laboratory classes have traditionally suffered from tremendous variability of instruction. In large first year classes there will almost always be many lab sections with many different lab instructors, which compounds the problem. Students have identified laboratory instruction as a consistent weakness in RMIT's Engineering program.

As a result of the above, the authors felt that it was appropriate to undertake a pilot project to determine the viability of lab video production. A series of six videos were produced for this purpose with the intention of providing a general introduction to the labs as opposed to a week 
by week supplementation of the lab material covered; the intention being that the latter would follow if the former were successful.

\section{Rationale}

Typically the electronics course has enrolments of 250 students or more and since the laboratory facility is designed to accommodate a maximum 24 students, an enrolment of 250 necessitates 11 lab sections. With this number of lab sessions, it is not feasible for the lecturer to take all of the sections. Hence graduate students are often used as laboratory instructors, partly because of the need to provide them with employment opportunities to support their studies and also because some may seek to pursue academic careers and it is felt that the labs provide a viable training opportunity for them. These students frequently have very little, if any, previous teaching experience and although they may know the subject matter well, they cannot necessarily teach it well. A less than perfect command of the English language in respect of international graduate students can also be a factor.

A common scenario is that 7/8 different graduate students are engaged to teach approximately 11 lab sections and the combination of factors outlined above frequently means that there is considerable variability in teaching quality and thus in the educational experience of the students. The lecture and laboratory sections are closely interwoven, which helps to alleviate some but by no means all of the issues and it should be noted that labs, from a teaching point of view, are often very demanding even for the most experienced instructor.

The motivation behind development of quasi interactive videos for the labs was thus to provide the students with the opportunity to gain a uniform and consistent understanding of the overall material to be covered, to understand the lab environment and the correct use of equipment and to gain the knowledge and ability to construct circuits, make measurements and trouble-shoot.

The videos also serve to help prepare the laboratory instructors for the lab sessions by refreshing their knowledge of the overall material to be covered, by demonstrating an appropriate laboratory teaching technique and by laying the ground rules for students in terms of lab safety and appropriate use of equipment.

By viewing the videos ahead of time the students can be better prepared, can clearly see the link between theory and practice and have the opportunity to overcome some of the deficiencies and inequalities that may exist in the lab instruction. However, in contrast to the series of lecture videos previously produced and as previously stated, the lab videos were not intended to supplement the specific lab material on a week by week basis.

Structure of the Electronics Laboratory

- The Electronics course is run over one semester and there are a total of 13 labs each of which is two hours long;

- Students work in groups of two and are expected to keep a work book, which is reviewed by the lab instructors after each lab session;

- There is an individual one hour lab exam in the fourteenth week; 
- The first six labs are analog, five of which are completed during one two-hour lab period and one of which runs over two weeks;

- The laboratories are assessed by way of demonstration and also via material entered into the students' work books. For example, if an experiment requires the design of an amplifier with a voltage gain of 50, students would demonstrate to the laboratory instructor the input and output wave form on an oscilloscope after completing the construction and testing;

- Students are expected to have prepared for the lab by way of preliminary exercises. Oneweek labs tend to be very busy and ill prepared students are unlikely to be able to finish in the allotted time;

- The second half of the semester is focused on digital electronics and students check out a breadboard and components to complete three digital projects. They are encouraged to work outside of normal lab time and come to the scheduled lab for questions and demonstration of their projects.

\section{Video Production Methods}

The goal was to make the videos look as professional as possible and to maintain an interactive feel to the presentation. This was achieved by not merely filming lab sessions in progress but by making each video a production - scripting, filming and editing to produce a product that makes the student feel that he or she is actively engaging with the presenter. Each video covers certain material and then pauses, suggesting to the students that they do certain exercises or refer to certain material. In this way the videos speak to the students and produce the quasi interactive approach that students seem to respond to well.

Pre-Production of the videos involved choosing the appropriate location and building a cart which duplicated the equipment and facilities found in the laboratory. It was important to film mainly in a studio in order to control the lighting appropriately, vary the sound as required and produce a professional product. The material to be presented was carefully planned and story boards were developed.

Actual production of the videos involved some shots in the lab but mostly filming in the studio. It was important to create a spontaneous and natural feel to the videos and thus the skills of the presenter were critical ${ }^{[2]}$. Two cameras were used to enable shots from different angles to be taken.

Post production involved editing and packaging the videos. Editing was undertaken using Pinnacle Studio ${ }^{[3]}$.

\section{Student/Laboratory Instructor Feedback}

A total of six lab videos were produced but due to a shortage of time between the video production and the actual lab sessions, only five were distributed to the students. It was considered very important to gain meaningful feedback on the value of the videos and therefore questionnaires were distributed to gather responses for evaluation. Students were divided into three groups for this purpose - those who watched the videos before attending the actual lab 
sessions, those students who watched the videos after attending the sessions and those who chose not to view the videos at all.

Responses from the students who watched the lab videos before attending the lab sessions were evaluated on the basis of the following questions/statements:

- How helpful were the videos in preparing you for the labs?

- The lab videos are an excellent teaching and learning tool

- I would like videos produced for all labs in this course

- I would like videos produced for other lab courses

- The material was easier to follow from the videos than from the face to face lab sessions.

Responses from the students who watched the lab videos after attending the lab sessions were evaluated on the basis of the following questions/statements:

- Did you gain a better understanding of the labs after viewing the videos?

- Do you feel that the videos would have helped you more if you had watched them before attending lab sessions?

- The lab videos are an excellent teaching and learning tool;

- I would like videos produced for all labs in this course;

- I would like videos produced for other lab courses;

- The material was easier to follow from the videos than from the face to face lab sessions

39 responses were received from a sample of 75 students and the overall feedback from the first year students was overwhelmingly positive. The results were calculated as an average score out of 5 for each statement, ordered from the highest to the lowest score.

Students who watched the videos before attending the lab sessions (Refer Figure 1)

High scores were obtained in response to the question "How helpful were the videos in preparing you for the labs" and also the statement "The lab videos are an excellent teaching and learning tool" This feedback was encouraging for the authors. The related statement "I would like videos produced for other lab courses" also scored well. The next highest score related to the statement "The material was easier to follow from the videos than from the face to face lab sessions." The vast majority of students indicated that they used the videos before the lab sessions, so as to understand what they were doing in terms of experimental procedures. A significant number of these students also felt the videos were useful for review purposes after the labs.

Students who watched the videos after attending the lab sessions (Refer Figure 2)

High scores were obtained in response to the question "Did you gain a better understanding of the labs after viewing the videos? High scores were also obtained in respect of the statement "Videos are an excellent teaching and learning tool". The related question about videos being produced for other lab courses" also scored well; as did the statement "The material was easier to follow from the videos than from the face to face lab sessions. 
Responses from the laboratory instructors

In addition to the above, responses from lab instructors were also gathered. They found videos to be an excellent preparation and teaching tool and valued their role in providing them with the opportunity for personal revision before teaching the lab sessions. Instructors commented on the fact that students can review the lab material at any time, either after a couple of classes, when they may be more comfortable with the labs \& equipment or later for use in understanding more complex experiments.

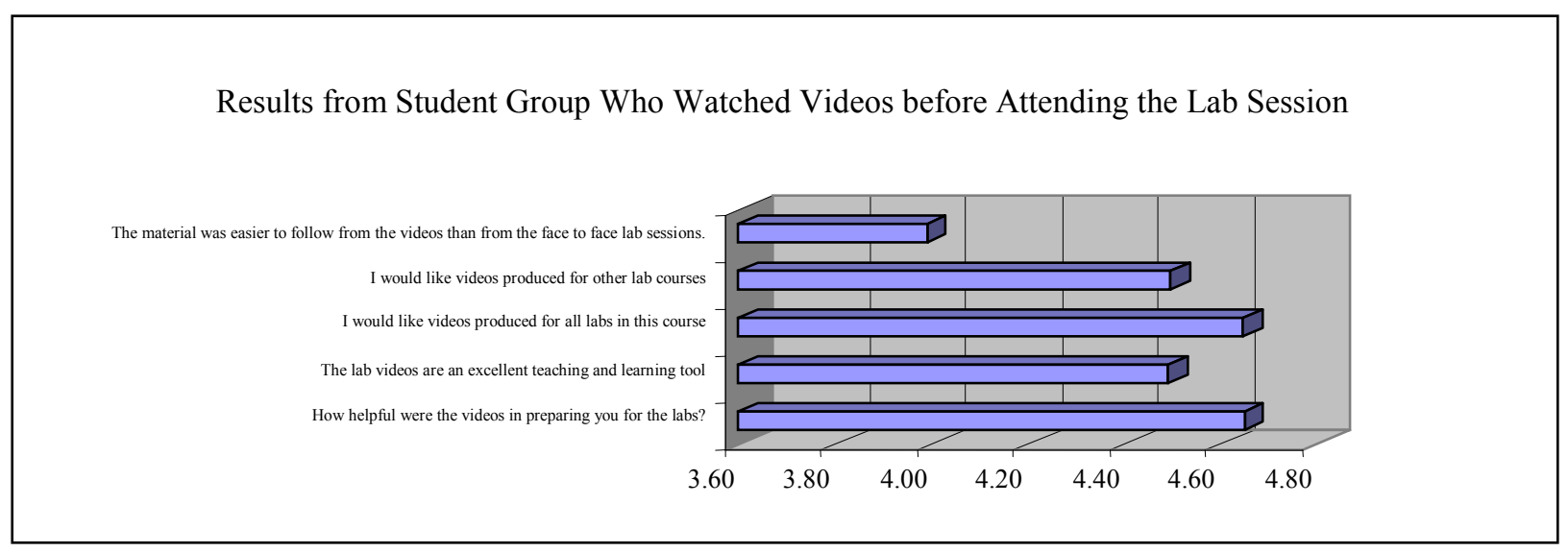

Figure 1 Average score against each statement from the student group who watched the videos before attending the lab session from 'Highest scale of 5 and lowest score of 0 '.

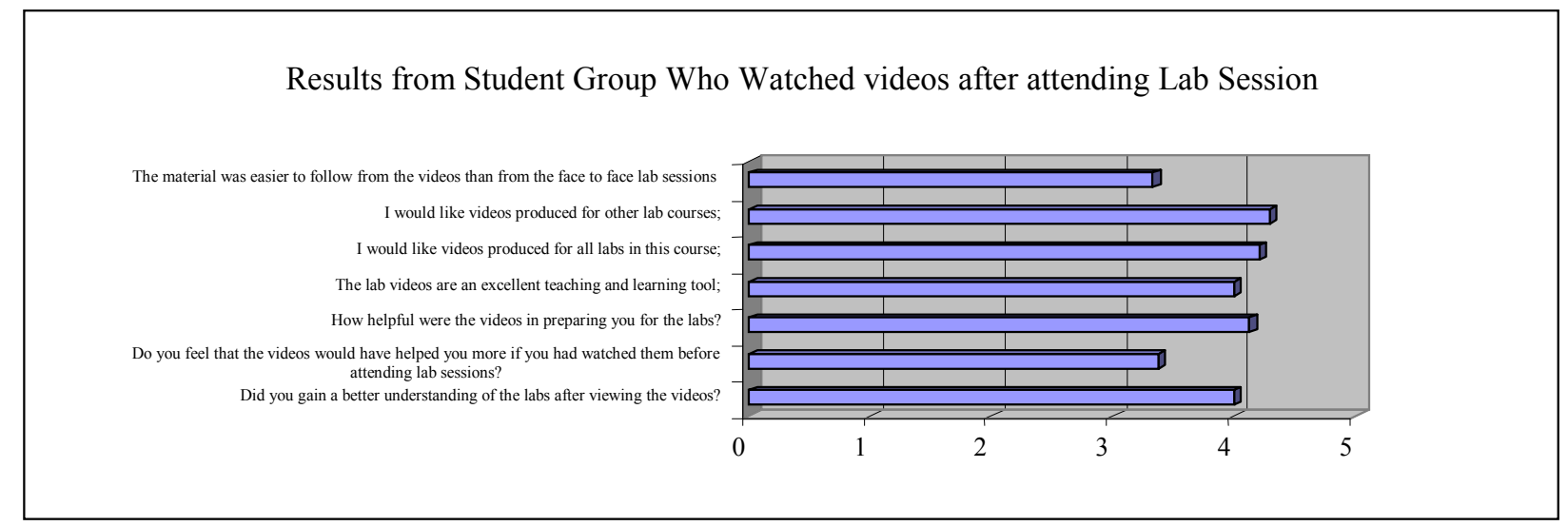

Figure 2 Average score against each statement from the student group who watched the videos before attending the lab session from 'Highest scale of 5 and lowest score of 0 '.

\section{Benefits/Advantages of Lab Video Production}

As previously stated there are many benefits and advantages in producing quasi interactive videos to supplement laboratory teaching in first year electronics, as well as other courses. These benefits include: 
- the opportunity to provide more uniform quality and consistency across all lab sections;

- the opportunity to save time during tightly scheduled lab sessions by preparing students ahead of time;

- confidence on the part of the institution that all occupational health and safety issues have been adequately covered;

- use as a revision tool for tests and exams;

- the opportunities for use in distance education. This has the potential to provide a service to students in remote locations, which is particularly important in countries such as Australia, and also to provide a valuable opportunity for the institution to earn external revenue, which is so important in the current university funding environment.

The authors are not aware of any disadvantages inherent in the use of quasi interactive videos, particularly since they are a supplement to and not an alternative means of instruction. Ample facilities are provided for reviewing the videos at RMIT if students do not have adequate facilities at home.

\section{Discussion/Conclusion}

The authors recognise that the feedback obtained does not constitute valid data in an empirical sense but the purpose was to confirm what the authors already believed to be the case, that quasi interactive videos can be a viable and valuable resource for lab sessions as well as for lectures. More valid data could and will be obtained by looking at the final results of the three groups of students - those who generally watched the videos prior to lab sessions, those who generally watched them after the lab sessions and those who chose not to view them at all. However, the authors are sufficiently confident, from the questionnaire responses, comments and general feedback received from students, laboratory instructors, staff and others to consider this pilot project a success and to move into full scale production of lab videos on a session by session basis at the earliest opportunity.

References

1. Burton P.C.M., “Quasi Interactive Video Production as a Teaching, Marketing and Retention Tool," Proceedings of the 2005 American Society of Engineering Education Annual Conference and Exposition.

2. Bauman, E.J., Jurgens D.E., “Undergraduate Electrical Engineering via Tutored Video Instruction,” Frontiers in Education Conference, 1994, pp 460-463.

3. Long, B., Schenk S., "The Digital Filmmaking Handbook,” Charles River Media, 2002 